\title{
Os benefícios da massagem terapêutica na gravidez e trabalho de parto
}

\author{
Ana Filipa Serafim Tralhão ${ }^{1}$ \\ The benefits of massage therapy in pregnancy and labor \\ Dora Honorato Carteiro \\ Los beneficios de la terapia de masaje durante el embarazo y
el parto
}

DOI: https://doi.org/10.53795/rapeo.v21.2021.11

\section{RESUMO}

A gravidez e o trabalho de parto implicam adaptações e desconfortos tais como a presença de alguma dor. A promoção do conforto ao longo do ciclo gravídico e trabalho de parto passa pelo controlo da dor e é determinante para uma vivência positiva atual e em futuros projetos de maternidade. A utilização da massagem terapêutica deve ser valorizada nos cuidados à grávida/casal.

Objetivo: Identificar os benefícios da massagem terapêutica na gravidez e trabalho de parto.

Método: Revisão sistemática da literatura, utilizando o método PI[C]OD, a questão orientadora para a revisão foi "Quais os benefícios da massagem terapêutica durante a gravidez e trabalho de parto?". A pesquisa foi realizada nas plataformas B-On e EBSCOhost (Medline complete, Nursing \& Allied Health Collection: Comprehensive, Medic Latina, CINAHL Complete), com um friso temporal de 8 anos, utilizando os descritores massage, therapy e pregnant woman.

Resultados: Foram selecionados 11 artigos que analisam a utilização da massagem terapêutica como intervenção eficaz na gravidez e trabalho de parto. A evidência mostra os benefícios desta no alívio dos desconfortos da gravidez e na redução da dor e da duração do trabalho de parto.

Conclusão: A massagem é uma técnica de relaxamento que apresenta benefícios para a díade: grávida-feto. A inclusão desta intervenção na prática de cuidados durante a gravidez e trabalho de parto é pertinente, acessível, segura, autónoma e facilmente aceite pelas grávidas.

Palavras-chave: grávida; massagem; terapia; trabalho de parto

Abstract: Pregnancy and labor involve adaptations and discomfort such as the presence of some pain. The promotion of comfort throughout the pregnancy and labor involves pain control and is crucial for a positive experience of the current pregnancy and future maternity projects. The use of therapeutic massage should be valued in the care of pregnant women/couples.

Objective: Identify the benefits of massage therapy in pregnancy and labor.

Method: A systematic literature review using the PI[C]OD method was defined as the question: "What are the benefits of therapeutic massage during pregnancy and labor?". The research was carried out on the B-On and EBSCOhost platforms (Medline complete, Nursing \& Allied Health Collection: Comprehensive, Medic Latina, CINAHL Complete), with a time frame of 8 years, using the descriptors massage, therapy and pregnant woman.

Results: 11 articles were selected that analyze the use of therapeutic massage as an effective intervention in pregnancy and labor. Evidence shows its benefits in relieving the discomforts of pregnancy and reducing the pain and duration of labor.

Conclusion: Massage is a relaxation technique that has benefits for the dyad: pregnant-fetus. The inclusion of this intervention in care practices during pregnancy and labor is relevant, accessible, safe, autonomous and easily accepted by pregnant women.

Keywords: labor; massage; pregnant woman; therapy
Resumen: El embarazo y el trabajo de parto implican adaptaciones y molestias como la presencia dde dolor. La promoción de la comodidad durante todo el ciclo del embarazo y el parto implica el control del dolor y es fundamental para una experiencia positiva hoy y en futuros proyectos de maternidad. Se debe valorar el uso de masajes terapéuticos en el cuidado de mujeres/parejas embarazadas.

Objetivo: Identificar los beneficios de la terapia de masaje en el embarazo y el parto.

Método: Una revisión sistemática de la literatura, utilizando el método PI[C]OD, se definió como la pregunta: “¿Cuáles son los beneficios de la masoterapia durante el embarazo y el trabajo de parto?". La investigación se realizó en las plataformas B-On y EBSCOhost (Medline complete, Nursing \& Allied Health Collection: Comprehensive, Medic Latina, ClNAHL Complete), con un marco temporal de 8 años, utilizando los descriptores massage, therapy, pregnant woman. Resultados: Se seleccionaron artículos 11 que analizan el uso del masaje terapéutico como una intervención eficaz en el embarazo y el parto. La evidencia muestra sus beneficios para aliviar las molestias del embarazo y reducir el dolor y la duración del trabajo de parto.

Conclusión: El masaje es una técnica de relajación que tiene beneficios para la díada: embarazada-feto. La inclusión de esta intervención en las prácticas de atención durante el embarazo y el parto es relevante, accesible, segura, autónoma y de fácil aceptación por parte de la gestante.

Palabras Clave: masaje; mujeres embarazadas; terapia; trabajo de parto;

${ }_{1}^{1}$ Mestranda em Enfermagem de Saúde Materna e Obstétrica, Enfermeira no Hospital Vila Franca de Xira

${ }_{2}$ PhD, Professora Adjunta, Escola Superior de Saúde da Cruz Vermelha Portuguesa 


\section{Introdução}

A gravidez é um processo fisiológico que permite o desenvolvimento fetal e implica adaptações de comportamentos e hábitos na vida da mulher. A transição para a maternidade envolve alterações ao nível físico e psicológico, que podem assumir um carácter patológico ${ }^{(1)}$.

Durante a gravidez ocorrem alterações na postura corporal, nomeadamente a anteversão pélvica, o aumento da lordose, o aumento do peso corporal e a mudança do centro de gravidade. O conjunto destes fatores perturba o equilíbrio, reduzindo a mobilidade das articulações sacroilíacas e obrigando o corpo a compensar com outras estruturas ósseas, como as articulações do quadril e membros inferiores. Estas adaptações corporais provocam alterações na disposição dos ossos e, consequentemente, o aumento de tensão nos tecidos moles da pélvis ${ }^{(2)}$. $\mathrm{O}$ aumento gradual de stress, durante a gravidez, é também um fator de risco para o bem-estar materno-fetal, aumenta a probabilidade de depressão perinatal, hipertensão induzida pela gravidez, pré-eclâmpsia, parto pré-termo e baixo peso do recém-nascido ${ }^{(4)}$.

A dor de trabalho de parto inicia-se com a compreensão do estímulo nociceptivo, que é recebido a nível central, e ao qual a grávida atribui o significado de dor ${ }^{(5)}$. A forma como a mulher perceciona esta dor depende da sua experiência de vida, cultura, contexto social, espiritual, o que a torna um fenómeno constituído por interpretações sensoriais e afetivas individuais e complexas, que consequentemente varia de indivíduo para indivíduo ${ }^{(1,5,6)}$. No primeiro e segundo estadios de trabalho de parto a dor é predominantemente visceral, com o estímulo nociceptivo provocado pela distensão do segmento inferior do útero e colo e pela contratilidade miometral ${ }^{(5,6)}$.

O controlo da dor, durante o trabalho de parto é importante na medida em que, as vivências positivas e negativas vão influenciar próximas gravidezes e partos. $\mathrm{O}$ alívio da dor promove a tranquilidade que a mulher/casal necessitam para vivenciar esta experiência única, a maternidade/paternidade ${ }^{(7)}$. A mulher tem necessidade de controlar o corpo, o trabalho de parto e a dor a ele associada e, por esse motivo, a procura por estratégias alternativas no controlo da dor tem aumentado(6). Podemos constatar esta necessidade de controlo da dor nomeadamente no Brasil, onde os estudos mostram que as mulheres apresentam preferência por partos por cesariana por este receio da dor ${ }^{(1)}$. 
A duração do trabalho de parto é um dos fatores com mais influência no bem-estar materno e fetal, aumenta o risco de complicações neonatais graves como morte fetal ou neonatal, asfixia, infeção e alterações neurológicas ou físicas. A evidência científica também expõe algumas complicações maternas do prolongamento do trabalho de parto como a hemorragia pós-parto, infeção e alterações psicológicas provocadas pela ansiedade, falta de sono e fadiga ${ }^{(3)}$.

Neste sentido surge cada vez mais a necessidade de adotar estratégias que minimizem este desconforto, nomeadamente com estratégias da medicina alternativa. A Organização Mundial de Saúde define medicina complementar e alternativa (CAM) como um conjunto de práticas de cuidados de saúde, que não fazem parte da tradição ou da medicina convencional de determinado país e que não estão totalmente integradas no sistema de cuidados de saúde dominante. Em alguns países, as CAM são utilizadas em simultâneo ou como complemento da prática tradicional( ${ }^{(8)}$. O termo complementar refere-se a práticas não usadas convencionalmente, enquanto alternativa às abordagens usadas em vez das práticas convencionais. Medicamentos e terapias alternativas complementares são conhecidas como abordagens de saúde que incluem várias modalidades para promover, tratar e manter o bem-estar e a saúde ${ }^{(9)}$.

Assim, a esfera de ação das CAM é ampla e variada, abrange diversas terapias com vastas abordagens como a acupuntura, a aromoterapia, hidroterapia, massoterapia, entre outras. As evidências que apoiam as CAM relativamente à eficácia, eficiência ou segurança são menores quando comparadas com medicamentos licenciados convencionais, mostrando a necessidade de mais investigação na área(10,11).

Neste estudo foi selecionada a utilização da massagem terapêutica como área de investigação a desenvolver, por ser uma área de interesse pessoal e integrar uma medida não farmacológica, acessível, para alívio da dor e de outros desconfortos, durante a gravidez e trabalho de parto, nomeadamente pelos profissionais de saúde. Foi definido como objetivo identificar os benefícios da massagem terapêutica na gravidez e trabalho de parto.

\section{Método}

Como ponto de partida para a revisão sistemática da literatura, considerando a temática e a sua conceptualização teórica foi formulada a questão de revisão, de acordo com a metodologia PI[C]OD: "Quais os benefícios da massagem terapêutica durante a gravidez e 
trabalho de parto". Foram definidos os critérios de inclusão e exclusão e o objetivo de identificar os benefícios da massagem terapêutica na grávida, como consta na tabela 1.

As palavras-chave que foram hierarquizadas para a pesquisa foram: massagem, terapia, grávida e trabalho de parto.

Tabela 1. Critérios de inclusão, exclusão, palavras-chave e expressão de pesquisa

Critérios de inclusão

P Participantes

(P1) Grávidas

(P2) Profissionais de saúde que prestam cuidados a grávidas

I Intervenções Artigos com intervenção de massagem terapêutica na vigilância da gravidez e/ou trabalho de parto

[C] Comparações

- Outcomes

D Desenho
Benefícios da massagem terapêutica durante a gravidez e/ou trabalho de parto

-Estudos primários -Revisões sistemáticas da literatura

-Quantitativo / qualitativo

\section{Critérios de exclusão}

Grávidas de alto risco, homens, crianças, mulheres não grávidas

Artigos que não se enquadrem com a temática massagem terapêutica

Artigos que apresentem resultados da massagem terapêutica na mulher não grávida, homem ou criança Artigos sem caráter científico

\section{EXPRESSÃO DE PESQUISA}

\section{Massage $^{*}$ and therapy and pregnant woman}

A pesquisa foi realizada em Coimbra no dia 5.11.2021, nas bases de dados científicas das plataformas B-On e EBSCOhost (Medline complete, Nursing \& Allied Health Collection: Comprehensive, Medic Latina, CINAHL Complete), com a expressão de pesquisa, respetivamente: "massage* and therapy and pregnant woman". Foram definidos como limitadores gerais, o acesso a texto integral, resumo disponível, idiomas: inglês, português ou espanhol, data de publicação entre 2014.01.01 e 2021.10.31. Optou-se por um maior friso temporal para uma maior abrangência de estudos.

Nesta etapa, foi identificado como obstáculo a definição dos descritores a utilizar na pesquisa. Optou-se por utilizar a expressão massage therapy, que conduziu a resultados pouco significativos. A sua utilização separada, enquanto descritores contribuiu para a identificação de documentos científicos relevantes. O termo "therapy" não é considerado um descritor MeSH, contudo integra os entry term do descritor MeSH "therapeutics", cuja inclusão 
da expressão de pesquisa conduziu à ausência de resultados. O mesmo se verificou com a tentativa de incluir "labor". Assim, a pesquisa foi realizada com a expressão "massage*" and "therapy" and "pregnant woman".

Após a pesquisa de artigos, na fase de identificação do prisma, foram identificados 43 artigos nas bases de dados científicas, sendo que foram removidos 9 artigos duplicados. $\mathrm{Na}$ fase de seleção dos 34 artigos foram excluídos 17 artigos após leitura do título e resumo, por não cumprirem o critério de idiomas definidos, não existir referência à massagem terapêutica e apresentarem intervenções de massagem a grávidas de alto risco. Após a leitura integral dos 17 artigos, na fase de elegibilidade, foram excluídos 6 artigos por apresentarem terapias não farmacológicas que não incluíam a massagem ou aplicarem a massagem em mulheres que não vivenciavam a gravidez. Na fase de inclusão foram incluídos 5 artigos de âmbito qualitativo e 6 quantitativos (Figura 1).

Figura 1 - Prisma

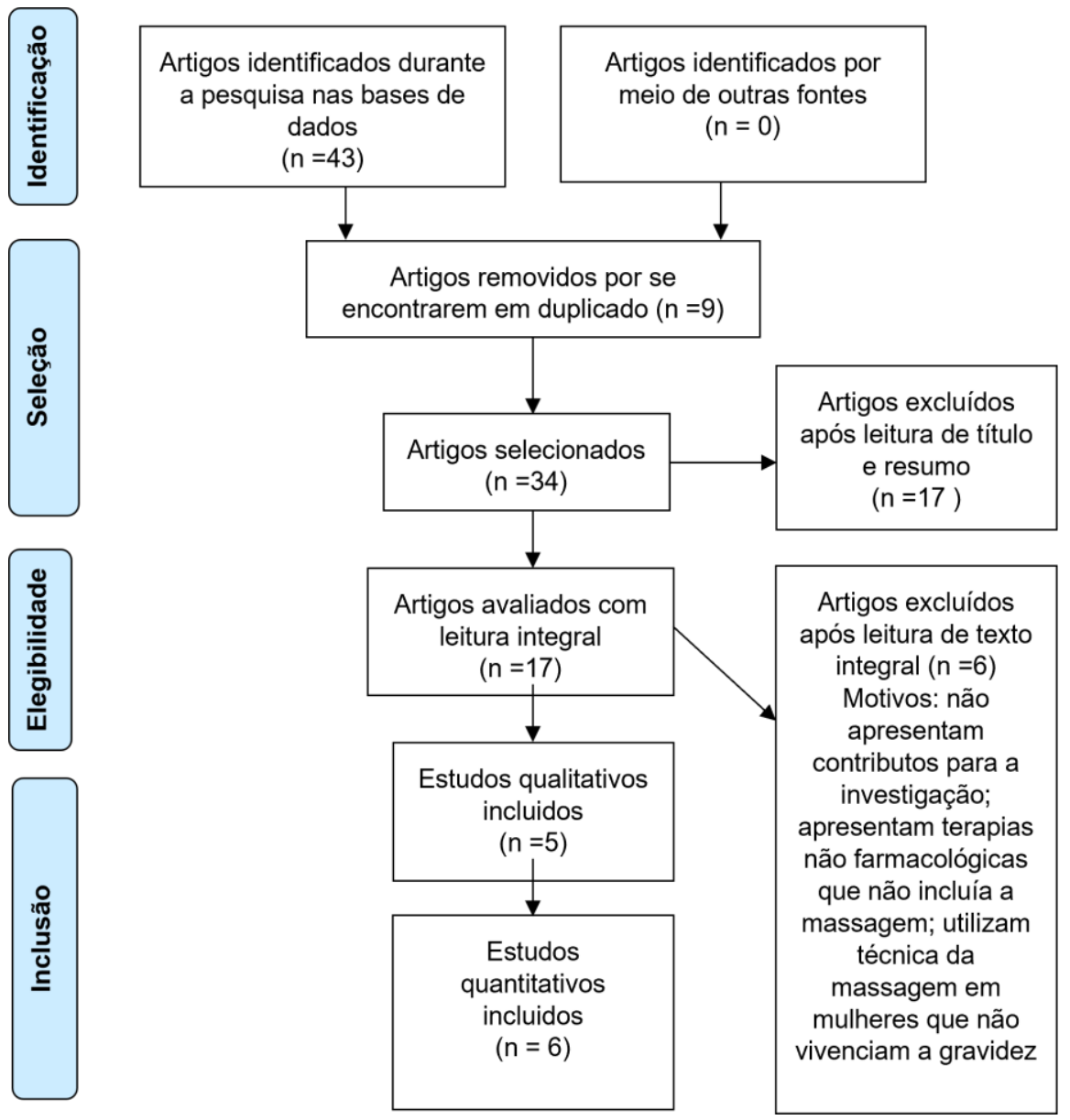




\section{Apresentação e Discussão dos resultados}

Na tabela 2, observa-se uma breve caraterização dos artigos incluídos nesta revisão sistemática da literatura, relativamente ao título, autores, tipo de estudo, participantes e objetivo dos respetivos estudos.

Tabela 2 - Caracterização dos artigos selecionados

\begin{tabular}{|l|}
\hline \multicolumn{1}{|c|}{ Título/ Autor/Ano } \\
\hline (1) Avaliação do impacto de um protocolo fisioterapêu- \\
tico na diminuição do quadro álgico durante a primeira \\
fase do trabalho de parto vaginal ${ }^{1}$ \\
Cardozo C, Cunha F (2018).
\end{tabular}

(2) The use of tensegrity massage in pregnant woman: a case report ${ }^{2}$

Wilk I, Kassolik K, Andrezejewski W, Kolodynska G, Forysiak E, Nowak B, Kurpas D (2019).

(3) Effect of Massage Therapy on Duration of Labour: A Randomized Controlled Trial ${ }^{3}$

Bolbol-Haghighi N, Masoumi S, Kazemi F (2016).

(4) Effects of Aromatherapy Massage on Pregnant Women's Stress and Immune Function: A Longitudinal, Prospective, Randomized Controlled Trial ${ }^{4}$

Chen P, Chou C, Yang L, Tsai Y, Chang Y, Liaw J (2017).

(5) Recursos não farmacológicos: atuação da fisioterapia no trabalho de parto, uma revisão sistemática ${ }^{5}$

Angelo P, Ribeiro K, Lins L, Rosendo A, Sousa V, Micussi M (2016).

(6) Women's use of complementary and alternative medicines during pregnancy: A cross-sectional study ${ }^{10}$

Hall H, Jolly K (2014).

(7) Healthcare professional views and experiences of complementary and alternative therapies in obstetric practice in Northeast Scotland: a prospective questionnaire survey ${ }^{11}$

Stewart D, Pallivalappila A, Shetty A, Pande B, McLay J (2014).

(8) Association of complementary and alternative therapies with mental health outcomes in pregnant women living in a postdisaster recovery environment ${ }^{12}$

Mendoza V, Harville E, Savage J, Giarratano G (2016).

(9) The effect of complementary medicines and therapies on maternal anxiety and depression in pregnancy: A systematic review and meta-analysis ${ }^{13}$

Smith C, Shewamene Z, Galbally M, Schmied V, Dahlen $\mathrm{H}$ (2018).

(10) Use of pharmacological and non-pharmacological labour pain management techniques and their relationship to maternal and infant birth outcomes: Examination of a nationally representative sample of 1835 pregnant women $^{14}$

Adams J, Frawley J, Steel A, Broom A, Sibbritt D (2015). (11) Terapias complementarias durante la gestación y parto. Revisión integrativa ${ }^{15}$

Bocanegra B, Sosa J, Simbaqueba D (2020).

\section{Tipo de estudo/Objetivo}

Estudo aleatório e randomizado (50 grávidas) Objetivo: Avaliar a eficiência de um protocolo fisioterapêutico na redução da dor na primeira fase de trabalho de parto e na humanização do parto vaginal.

Estudo observacional (2 grávidas)

Objetivo: Determinar a eficácia da massagem de tensão, combinada com a manipulação pontual de músculos específicos em grávidas. Estudo clínico randomizado (100 grávidas) Objetivo: Avaliar o efeito da massagem terapêutica na duração do trabalho de parto

Estudo randomizado e controlado, prospetivo e longitudinal (52 grávidas)

Objetivo: Avaliar os efeitos da massagem com aromaterapia no stress e na função imunológica das mulheres durante a gravidez.

Revisão sistemática

Objetivo: Avaliar os efeitos de recursos fisioterapêuticos aplicados para o alívio da dor durante o trabalho de parto.

Estudo de corte transversal (315 grávidas/ puérperas)

Objetivo: Determinar a utilização de CAM durante a gravidez, as razões de uso, quem recomendou e as características das mulheres que recorreram a estas estratégias.

Estudo prospetivo (135 profissionais de saúde)

Objetivo: Investigar o uso de terapias alternativas e complementares por profissionais de saúde do Reino Unido envolvidos no cuidado a mulheres grávidas e identificar preditoreschave de utilização.

Estudo de corte transversal (402 grávidas)

Objetivo: Avaliar a influência de CAM em muIheres grávidas que vivem num ambiente de recuperação pós-desastre e a sua associação com sintomas de doenças mentais.

Revisão sistemática com meta-análise (1092 grávidas)

Objetivo: Avaliar a eficácia e segurança de medidas não farmacológicas na depressão e ansiedade durante a gravidez.

Estudo longitudinal (2445 grávidas ou puérperas)

Objetivo: Investigar a utilização de técnicas farmacológicas e não farmacológicas de gestão da dor do parto, por parte das mulheres.

\section{Revisão integrativa}

Objetivo: Descrever técnicas de CAM que contribuem para o bem-estar durante a gravidez e parto. 
A gravidez e a maternidade são transições na vida de uma mulher que podem gerar stress e desconforto. Os enfermeiros especialistas em saúde materna e obstétrica (EESMO) são profissionais com a responsabilidade de promover uma transição saudável, planear intervenções em parceria com a mulher grávida e casal, com o objetivo de fortalecer a relação conjugal e parental, promovendo um ambiente conducente ao desenvolvimento e bem-estar materno e fetal.

O estudo de Stewart et al. ${ }^{(11)}$, investiga a utilização e recomendação de CAM's pelos profissionais de saúde numa maternidade da Escócia, recorrendo ao preenchimento de questionários pelos participantes. Como principais conclusões identifica-se que um terço dos profissionais de saúde recomendou a sua utilização para grávidas e os motivos nomeados para a não recomendação foram a falta de treino para executar a intervenção. Afirmam ainda que, o recurso a estas estratégias não farmacológicas é superior na Europa, nos Estados Unidos da América e na Austrália (11).

As terapias não farmacológicas não têm recetividade por todas as mulheres, isso devese ao facto de, não existirem estudos suficientes, ou os existentes, apresentarem amostras reduzidas $^{(10)}$. O estigma por parte da medicina convencional relativamente às CAM é, também, um obstáculo à mudança de mentalidade das grávidas. É importante reforçar que as CAM não impedem ou substituem a medicina convencional, mas que estas se podem complementar de forma a obter melhores resultados para um bem comum, o bem-estar materno e fetal ${ }^{(3,11)}$. Apesar do referido, Hall \& Jolly ${ }^{(10)}$ e Stewart et al. ${ }^{(11)}$ afirmam que a percentagem de mulheres que recorrem às CAM é superior, quando comparado com os homens tanto em situações de saúde como de doença. A sua utilização é diversa em tratamentos de fertilidade e na gravidez, com o objetivo de aliviar os sintomas associados a esta condição(10,11).

A massagem terapêutica é das quatro terapias mais utilizadas ${ }^{(5,14)}$ entendida como técnica de relaxamento que apresenta diversos benefícios para além de apresentar vantagens como ser acessível, segura e facilmente aceite pelas grávidas(3). Tem como princípio, a estimulação sensorial e manipulação dos tecidos moles do corpo, e é comumente usada para ajudar a relaxar os músculos tensos e acalmar o indivíduo, diminuindo os sintomas respiratórios. Promove ainda o contacto com o profissional, cujo objetivo é proporcionar relaxamento e aumentar o fluxo sanguíneo e a oxigenação ${ }^{(1,4,13)}$. Esta estratégia não farmacológica de alívio da dor apresenta benefícios ao nível da dor ${ }^{(1,5)}$ e relaxamento das parturientes ${ }^{(1)}$, pela redução da 
secreção de hormonas como a adrenalina e noradrenalina e o aumento de endorfinas e ocitocina ${ }^{(3)}$. A conjugação destes fatores influencia a duração do primeiro e segundo estadios de trabalho de parto, promovidos pelo aumento das contrações uterinas ${ }^{(3)}$, pela evolução favorável da dilatação do colo do útero e aumento da saturação de oxigénio ${ }^{1,}$

Durante a gravidez, a massagem, está indicada em queixas como edema ou dor nos membros inferiores, coexistentes com dor em áreas do corpo, como dor de pescoço, ombro ou dor sacro lombar. As contraindicações para a massagem em grávidas também estão evidenciadas, incluem um desvio à normalidade no decorrer da gravidez associado ao desenvolvimento fetal, disfunção do sistema urogenital; nefropatias: nefrocistite, nefrolitíase, insuficiência renal; doenças da tiróide: hipertiroidismo e hipotiroidismo, bem como hiperfunção e hipofunção endócrina; pancreatopatias (pancreatite, diabetes mellitus gestacional); nefropatia diabética; doenças cardiovasculares ${ }^{(2)}$.

O benefício da massagem terapêutica ficou comprovado através da intervenção de estudantes de obstetrícia submetidos a formação prévia(3). A inclusão de outros profissionais da equipa multidisciplinar, como fisioterapeutas, também foi considerada benéfica, atuando ao nível da comunicação entre a equipa e a família e no controlo do estado emocional e físico, através da realização se exercícios não invasivos promotores do conforto ${ }^{(1)}$.

Na vertente emocional, a depressão pós-parto é uma causa comum de morbilidade materna após o parto e pode conduzir a uma depressão crónica. A depressão e ansiedade na gravidez são frequentes e podem existir simultaneamente, cerca de $13 \%$ das mulheres vivenciam uma depressão major durante a gravidez, com prevalência crescente de até $22 \%$ nos primeiros doze meses após o parto. Os valores agravam-se quando se discute depressão pósparto em mães adolescentes ${ }^{(16,17)}$. Estas patologias têm um impacto significativo em todos os aspetos da vida da mulher, incluindo o feto e familiares que a rodeiam. São associadas a comportamentos inadequados no autocuidado materno e do recém-nascido, bem como a dificuldades sociais e de interação, prematuridade e baixo peso ao nascer. A criança poderá apresentar patologias no desenvolvimento neurológico, físico e psicológico, alterações de desenvolvimento de linguagem, comportamentos sociais e emocionais, concomitantes com um risco aumentado de depressão na idade adulta ${ }^{(13,16)}$.

A massagem terapêutica demonstra-se benéfica em mulheres que apresentam doença mental, como depressão e ansiedade, quando estudada a sua relação, confirma-se a redução da sintomatologia e prevalência( $(4,12,13)$. Contudo, Smith et al. ${ }^{(13)}$ salvaguardam os resultados 
referindo a escassez, na literatura, de estudos randomizados relevantes, que relacionem as CAM com a ansiedade e depressão. A aromoterapia, frequentemente utilizada, em simultâneo, com a massagem terapêutica pode reduzir o stress na gravidez ${ }^{(4)}$.

Apesar dos dados anteriormente referidos, quando a massagem é relacionada com o apoio social há referência à possível insignificância dos resultados obtidos anteriormente, isto é, as mulheres submetidas a massagem durante a gravidez, receberam o toque reconfortante com mais frequência de parceiros masculinos ou da própria mãe. Assim, os efeitos observados da massagem podem ser devidos ao aumento do apoio social( $\underline{12})$. O impacto positivo desta terapia permite a redução da prescrição de medicação para a depressão, que na gravidez, potencia risco para o feto, sendo que as grávidas e mães têm preferência pelo tratamento não farmacológico( ${ }^{(13)}$.

No trabalho de parto, as evidências demonstram vantagens na utilização da massagem terapêutica na duração e condução do trabalho de parto, na redução da dor, no tipo de parto e na melhoria da função respiratória. A duração do primeiro e segundo estadio do trabalho de parto foram significativamente mais curtos com a intervenção dos profissionais de saúde que recorreram a esta técnica. O recurso à massagem terapêutica intraparto, diminui o primeiro estadio do trabalho de parto e prepara o períneo para o segundo estadio, são realizados ensinos à parturiente sobre respiração, exercícios da musculatura das pélvis e as posições de alívio da dor, promovendo o parto eutócico ${ }^{(1,3)}$. Consequentemente, os níveis de saturação de oxigénio são influenciados positivamente, assim como a melhoria do quadro respiratório, prevenindo a hiperventilação e consequentemente a alcalose respiratória, o que possibilita maior perfusão placentária, redução do quadro álgico, ansiedade e stress ${ }^{(1)}$.

O tipo de parto é também influenciado pela massagem terapêutica, as parturientes submetidas a esta terapia não farmacológica, apresentam maior probabilidade em ter um parto eutócico, (3) ou distócico vaginal, assim como iniciar a amamentação precocemente e mantê-la pelo menos durante seis semanas, quando comparadas com mulheres que não utilizaram a massagem como terapia, submetidas a epidural ou à administração de petidina $(\underline{14})$.

A análise do partograma, realizada por Bolbol-Haghighi et al. (3) , apresentou, no grupo de teste, menos alterações negativas relativamente ao grupo de controlo, isto é, nas alterações anormais na frequência cardíaca do feto e na contratilidade. Assim, os resultados da escala de apgar dos recém-nascidos foram significativamente positivos ao primeiro e quinto 
minuto de vida, estes valores foram maiores quando as grávidas foram sujeitas a massagem terapêutica.

Destaca-se ainda a importância de avaliar a grávida antes de ser aplicada qualquer metodologia da massagem terapêutica, esta deve ser adaptada à utente com base nos seus principais sinais e sintomas, estado físico e mental atual e nas mudanças que ocorram durante a terapia(2). A massagem pode ser realizada por profissionais de saúde formados, pelos maridos ou por doulas, sendo que o efeito da massagem realizada por doulas é maior em relação à massagem realizada pelo marido, desconhecendo-se o motivo desta diferença( $\underline{3}$. Estes dados corroboram as pesquisas de Mollart et al. ${ }^{(18)}$, que evidenciam a necessidade de formação e treino na utilização de CAM, por parte dos EESMO/parteiras. Neste estudo, a massagem foi uma das quatro principais técnicas desenvolvidas pelos participantes, sendo que, os profissionais de saúde que desenvolveram formação especializada neste âmbito, possuem mais conhecimento e confiança para avaliação da grávida/ parturiente e aplicação das técnicas, bem como capacidade para discutir e interagir com as mulheres. Os autores destacam a necessidade e a importância de incluir a temática e o treino no plano de estudo dos cursos universitários, uma vez que, a qualidade da abordagem em ambiente académico foi considerada insuficiente, o que não permite aos profissionais desenvolverem as CAM de forma segura e suportada em evidência $(\underline{18})$.

\section{CONCLUSÃO}

A promoção da saúde e bem-estar materno e fetal, são a prioridade da prática dos EESMO, o recurso às CAM durante a gravidez e trabalho de parto, nomeadamente à massagem, é benéfico e referido pela Ordem dos Enfermeiros.

A massagem terapêutica é uma estratégia não farmacológica adequada às necessidades da grávida, por ser eficaz, não invasiva, segura, acessível e de baixo custo que produz efeitos terapêuticos positivos e necessários ao estado gravídico e ao trabalho de parto, prevenindo possíveis complicações associadas a estes períodos.

O EESMO é responsável por incluir e incentivar as terapias não farmacológicas durante a vigilância da gravidez e trabalho de parto, promovendo uma opção informada da grávida, a humanização do parto, a redução de terapias farmacológicas e a diminuição da prevalência de ansiedade e depressão pré e pós-natal. 
A massagem terapêutica pode ser associada a outras intervenções, tais como a aromoterapia, hidroterapia, cromoterapia, entre outras, a sua aplicabilidade difere entre grávidas e deve ser realizada sempre uma avaliação inicial antes de qualquer procedimento.

De acordo com os resultados obtidos conclui-se que a massagem terapêutica promove, durante o trabalho de parto, relaxamento das parturientes com indicadores positivos relativos à frequência cardíaca, saturação de oxigénio, dor, dilatação cervical, contratilidade, duração do trabalho de parto, tipo de parto e scores elevados na escala de apgar. A evidência científica comprova ainda a redução da prescrição de medicação, quando associada esta terapia.

Apesar de, já existirem evidências científicas relativamente ao uso de terapias alternativas e complementares na gravidez, estas não são ainda uma prática recorrente durante a gravidez e trabalho de parto. É necessária mudança global de mentalidades, quebrando estigmas relativos à sua utilização. O EESMO tem uma intervenção essencial em incentivar a utilização da massagem terapêutica, investir na formação para que sustente uma prática baseada na evidência, com recurso ao conhecimento e ao treino.

Cada vez mais se torna pertinente e emergente o investimento na continuidade de estudos nestas temáticas individualmente e em complementaridade, de forma a sustentar a evidência do benefício do seu uso.

\section{Referências}

1. Cardozo Cl, Cunha FM. Avaliação do impacto de um protocolo fisioterapêutico na diminuição do quadro álgico durante a primeira fase do trabalho de parto vaginal. Fisioter Bras [Internet]. 2019 Mai [citado em 5 nov. 2021];20(2):222-9. Disponível em: https://search.ebscohost.com/login.aspx?direct=true \&db=ccm\&AN=136258752\&lang=pt-br\&site=ehost-live

2. Wilk I, Kassolik K, Andrezejewski W, Kolodynska G, Forysiak E, Nowak B, Kurpas D. The use of tensegrity massage in pregnant woman: a case report. Med Sci Pulse [Internet]. 2019 Nov [citado em 5 nov. 2021];13(3):39-49. Disponível em: http://dx.doi.org/10.5604/01.3001.0013.5376

3. Bolbol-Haghighi N, Masoumi SZ, Kazemi F. Effect of Massage Therapy on Duration of Labour: a randomized Controlled Trial. J Clin Diagn Res [Internet]. 2016 Abr [citado em 5 nov. 2021];10(4):12-5. Disponível em: https://doi.org/10.7860/JCDR/2016/17447.7688

4. Chen P, Chou C, Yang L, Tsai Y, Chang Y, Liaw, J. Effects of aromatherapy massage on pregnant women's stress and immune function: a longitudinal, prospective, randomized controlled trial. J Altern Complement 
Med [Internet]. 2017 Out [citado em 5 nov. 2021];23(10):778-86. Disponível em: https://doi.org/10.1089/acm.2016.0426

5. Angelo PH, Ribeiro KC, Lins LG, Rosendo AM, Sousa VP, Micussi MT. Recursos não farmacológicos: atuação da fisioterapia no trabalho de parto, uma revisão sistemática. Fisioter Bras [Internet]. 2016 Out [citado em 5 nov. 2021];17(3), 285 -92. Disponível em https://search.ebscohost.com/login.aspx?di$\underline{\text { rect=true } \& \mathrm{db}=\mathrm{ccm} \& \mathrm{AN}=125930078 \& \text { lang=pt-br\&site=ehost-live }}$

6. Ferreira S. Métodos não farmacológicos de alívio da dor. In: Néné M, Marques R, Batista MA, editores. Enfermagem de Saúde Materna e Obstétrica. 1ํedição. Lisboa: Lidel; 2016. p. 416-24.

7. Guerra A. Analgesia e anestesia em obstetrícia. In: Néné M, Marques R, Batista MA, editores. Enfermagem de Saúde Materna e Obstétrica. 1ª edição. Lisboa: Lidel; 2016. p. 425-31.

8. World Health Organization. WHO global report on traditional and complementary medicine 2019 [Internet]. Genebra: World Health Organization; 2019 [citado 5 nov. 2021]. Disponível em https://apps.who.int/iris/handle/10665/312342

9. National Institutes of Health. National center for complementary and integrative health: Complementary, alternative or integrative health: what's in a name? [Internet]. Estados Unidos: National Institutes of Health; [atualizado em 2021 Abr; citado em 2021 nov] Disponível em https://nccih.nih.gov/health/integrative-health\#hed1

10. Hall HR, Jolly K. Women's use of complementary and alternative medicines during pregnancy: a crosssectional study. Midwifery [Internet]. 2014 Mai [citado em 5 Nov. 2021];30(5):499-505. Disponível em: https://doi.org/10.1016/j.midw.2013.06.001

11. Stewart D, Pallivalappila AR, Shetty A, Pande B, McLay JS. Healthcare professional views and experiences of complementary and alternative therapies in obstetric practice in North East Scotland: a prospective questionnaire survey. BJOG [Internet]. 2014 Fev [citado em 5 nov. 2021];121(8):1015-9. Disponível em: https://doi.org/10.1111/1471-0528.12618

12. Mendoza VB, Harville E, Savage J, Giarratano G. Association of complementary and alternative therapies with mental health outcomes in pregnant women living in a postdisaster recovery environment. J Holist Nurs [Internet]. 2016 Out [citado em 5 nov. 2021];34(3):259-70. Disponível em: https://doi.org/10.1177/0898010115609250

13. Smith CA, Shewamene Z, Galbally M, Schmied V, Dahlen H. The effect of complementary medicines and therapies on maternal anxiety and depression in pregnancy: a systematic review and meta-analysis. J Affect Disord [Internet]. 2019 Fev [citado em 5 nov. 2021];245:428-39. Disponível em: https://doi.org/10.1016/i.jad.2018.11.054 
14. Adams J, Frawley J, Steel A, Broom A, Sibbritt D. Use of pharmacological and non-pharmacological labour pain management techniques and their relationship to maternal and infant birth outcomes: examination of a nationally representative sample of 1835 pregnant women. Midwifery [Internet]. $2015 \mathrm{Abr}$ [citado em 5 nov. 2021];31(4):458-63. Disponível em https://doi.org/10.1016/j.midw.2014.12.012

15. Bocanegra BM, Sosa JC, Simbaqueba DC. Terapias complementarias durante la gestación y parto. Revisión integrativa. Rev Cuid [Internet]. 2020 Mai [citado em 5 nov 2021];11(2)._Disponível em https://doi.org/10.15649/cuidarte.1056

16. Sangsawang B, Wacharasin C, Sangsawnag N. Interventions for the prevention of postpartum depression in adolescent mothers: a systematic review. Arch Womens Ment Health [Internet]. 2019 Ago [citado em 5 nov. 2021];22:215-28. Disponível em: https://doi.org/10.1007/s00737-018-0901-7

17. Wisner KL, Sit DK, McShea MC, Rizzo DM, Zoretich RA, Hughes CL, Eng HF, Luther JF, Wisniewski SR, Costantino ML, Confer AL, Moses-Kolko EL, Famy CS, Hanusa BH. Onset timing, thoughts of self-harm, and diagnoses in postpartum women with screen-positive depression findings. JAMA Psychiatry [Internet]. 2013 Mai [citado em 5 nov. 2021];70(5):490-8. Disponível em: https://doi.org/10.1001/jamapsychia$\underline{\operatorname{try} .2013 .87}$

18. Mollart L, Stulz V, Foureur M. Midwives knowledge and education/training in complementary and alternative medicine (CAM): A national survey. Elsevier [internet]. 2021 Ago [citado em 5 nov 2021];45. Disponível em: https://doi.org/10.1016/j.ctcp.2021.101473 